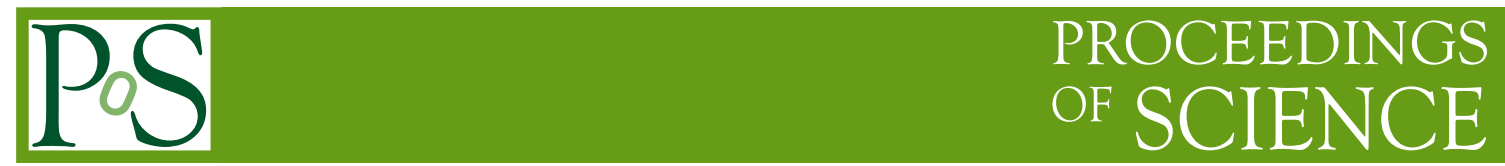

\title{
Searches for high energy neutrinos.
}

\author{
Jürgen Brunner* \\ Centre de Physique de Particule de Marseille \\ E-mail: juergen.brunnerdcern.ch
}

IceCube and ANTARES are neutrino detectors sensitive to energies from $20 \mathrm{GeV}$ up to PeV. Both detectors have been completed and take data. Several years of data have been already analysed including periods with the partly assembled detectors. The primary goal of these two neutrino telescopes is the observation of astrophysical sources of neutrinos. Results from searches for such neutrinos with different strategies will be presented. Atmospheric neutrinos consist an irreducible background for such searches. But they are an interesting study object by themselves.

The XIth International Conference on Heavy Quarks and Leptons

11-15 June, 2012

Prague, The Czech Republic

${ }^{*}$ Speaker. 


\section{Introduction}

More than one hundred years after the first observations of cosmic rays, and in spite of the impressive amount of data that have in the meantime been collected, many of the problems connected with their origin and propagation remain unsolved. It is a common, but poorly supported, belief that they must originate from catastrophic events which take place in our as well as in other Galaxies. Events such as Supernova explosions, Active Galactic Nuclei, Quasars and Micro Quasars, which are likely sources of high energy cosmic rays and gamma rays, could be intense neutrino sources as well. The measurement of the arrival direction and energy of such neutrinos, that would clarify the production mechanisms of high energy hadrons and gammas, requires very massive targets, of sizes far beyond those of present, conventional underground detectors. A possible solution, suggested over 20 years ago, is the use of water or ice as a Cherenkov target-detector. The prove of this concept has been performed in lake Baikal with the unambiguous detection of atmospheric neutrinos in the NT-96 detector [1]. Today two large detectors, ANTARES in the Mediterranean Sea and IceCube at the South pole take data and produce physics results. These two projects will be presented in the following.

\section{ANTARES}

A detailed description of the ANTARES detector can be found in [2]. The detector consists of 12 lines, equipped with photosensors, and a junction box which distributes the power and clock synchronization signals to the lines and collects the data. The junction box is connected to the shore by a $42 \mathrm{~km}$ electro-optical cable. The length of the detection lines is $450 \mathrm{~m}$, of which the lowest $100 \mathrm{~m}$ are not instrumented. Their horizontal separation is about $65 \mathrm{~m}$ and they are arranged to form a regular octagon on the sea floor. They are connected to the junction box with the help of a submarine using wet-mateable connectors. Each line comprises 25 storeys each separated by a vertical distance of $14.5 \mathrm{~m}$. The lines are kept taut by a buoy at the top of the line and an anchor on the seabed. The movement of the line elements due to the sea currents is continuously measured by an acoustic calibration system with an accuracy of $10 \mathrm{~cm} \mathrm{[3].}$

Each storey contains three $45^{\circ}$ downward-looking 10" photomultiplier tubes (PMT) inside pressure resistant glass spheres - the optical modules [4]. Some of the storeys contain supplementary calibration equipment such as acoustic hydrophones or optical beacons [5].

The signals of each photomultiplier are readout by two ASICs. The charges and arrival times of the PMT signals are digitised and stored for transfer to the shore station [6]. The time stamps are synchronised by a clock signal which is sent at regular intervals from the shore to all electronic cards. The overall time calibration is better than $0.5 \mathrm{~ns}$ [7]. Therefore the time resolution of the signal pulses is limited by the transit time spread of the photomultipliers $(\sigma \sim 1.3 \mathrm{~ns})$ [8] and by chromatic dispersion for distant light sources. All data are sent to the shore station. With the observed optical background rate of $70 \mathrm{kHz}$ per PMT at the single photon level this produces a data flow of several Gbit/s to the shore. In the shore station a PC farm performs a data filtering to reduce the data rate by at least a factor of 100 [9]. Several trigger algorithms are applied depending on the requested physics channel and on the observed optical noise. 
From March to December 2007 ANTARES operated in a 5-line configuration, followed by several months of operation with 10 installed detector lines. The detector construction was completed in May 2008.

\section{IceCube}

The IceCube detector [10] consists of 5,160 digital optical modules. Each of them contains a photomultiplier tube (identical to ANTARES), supporting hardware and electronics inside a pressure glass sphere. These optical modules are arranged on 86 strings frozen into the antarctic ice at depths from $1450 \mathrm{~m}$ to $2450 \mathrm{~m}$ with a spacing of $120 \mathrm{~m}$ between adjacent string, instrumenting one cubic kilometer. Optical properties of South Pole ice are depth dependent. Typical optical absorption lengths are 100-140 m and typical optical effective scattering lengths are 25-35 m. The latter limits the time resolution of the signal pulses from Cherenkov light to about $6 \mathrm{nsec}$, degrading thereby the ultimate angular resolution reachable from muon track reconstruction. Unlike sea water Antarctic ice has a low concentration in radioactive isotopes. In particular ${ }^{40} \mathrm{~K}$, abundant in salty sea water, is absent in ice. Further, no light-producing biological activity occurs. Therefore, the observed optical background rate at the single photon level is only $500 \mathrm{~Hz}$ per PMT in IceCube.

Beginning with the installation of the first string in 2005, IceCube has been operated in approximately year-long data taking seasons. During the antarctic summer seasons in December and January new strings had been deployed at a pace of about 20 strings per year. IceCube construction was finished in December 2010. From May 2008 to April 2009 IceCube operated with 40 installed strings, the so-called IC40 configuration. From May 2009 to April 201059 strings had been active (IC59). The results which will be presented in the following have been obtained from analysing these two data-taking periods.

\section{Neutrino Interactions: the cascade and the muon channel}

Neutrino interactions in the vicinity of the detector lead to two distinct signatures which are exploited in independent analyses. The charged current interaction of muon neutrinos

$$
v_{\mu}\left(\bar{v}_{\mu}\right)+N \rightarrow \mu^{\mp}+X
$$

and the charged current interaction of tau neutrinos with a subsequent muonic decay

$$
\begin{aligned}
v_{\tau}\left(\bar{v}_{\tau}\right)+N \rightarrow & \tau^{\mp}+X \\
& \tau^{\mp} \rightarrow \mu^{\mp}+\bar{v}_{\mu}\left(v_{\mu}\right)+v_{\tau}\left(\bar{v}_{\tau}\right)
\end{aligned}
$$

result both in a long muon track. At $\mathrm{TeV}$ and even more at $\mathrm{PeV}$ energies these muons have a range of several $\mathrm{km}$ in water or ice, largely exceeding the size of the detectors. The neutrino interaction vertex and the accompanying hadronic shower are outside the fiducial volume most of the time. The track signature yields a good angular resolution and ensures a clean separation of an upward going neutrino signal from the background of downward going atmospheric muons. The muon energy at the detector is deduced from the energy loss $\mathrm{dE} / \mathrm{dx}$ which is related to the "brightness" of the track in the detector. As the energy loss is mostly stochastic at $\mathrm{TeV} / \mathrm{PeV}$ energies, the 
muon energy can only be estimated with a precision of about a factor two. The calculation of the related neutrino energy depends on the assumed neutrino flux because the muon track has to be extrapolated upstream the detector to the neutrino vertex.

All other neutrino interactions as neutral current reactions

$$
v_{x}\left(\bar{v}_{x}\right)+N \rightarrow v_{x}\left(\bar{v}_{x}\right)+X
$$

electron neutrino charged current interactions

$$
v_{e}\left(\bar{v}_{e}\right)+N \rightarrow e^{\mp}+X
$$

and charged current interactions of tau neutrinos with a subsequent non-muonic decay

$$
\begin{aligned}
v_{\tau}\left(\bar{v}_{\tau}\right)+N \rightarrow & \tau^{\mp}+X \\
& \tau^{\mp} \rightarrow v_{\tau}\left(\bar{v}_{\tau}\right)+X
\end{aligned}
$$

lead to a so-called cascade signature with the exception of tau neutrino charged current interactions at energies above $10 \mathrm{PeV}$ which would yield two distinct cascades, one at the neutrino interaction vertex and a second one at the tau decay point. This special case is not considered in the following.

As a result of these neutrino interactions one obtains a hadronic and, depending on the channel, also an electromagnetic shower. They are very narrow and have a longitudinal extension of at most a few tens of meters. Due to the large spacing of adjacent detector elements in the coarsely equipped neutrino telescopes it is impossible to distinguish electromagnetic from hadronic showers. The observable signature in the detector is in all cases an isolated "cascade". Due to its small extension the angular resolution is worse here than for the track signature. This compromises the up/down separation. The main background for the cascade search comes from bright electromagnetic showers (e.g. due to bremsstrahlung) which accompany downward going atmospheric muons. The energy resolution for cascades can be better than for the track channel. All particles but the escaping neutrinos are seen in the detector and the brightness of the events correlates directly to the cascade energy which in turn is closely related to the neutrino energy. When using containment conditions for the neutrino vertex the power of the background rejection as well as the energy resolution can be further improved.

Whereas the effective volume for the cascade channel is close to the equipped volume of the detector, it is significantly larger for the muon channel due to the very long muon range.

\section{Atmospheric Neutrinos}

The major background for observing a TeV-scale diffuse neutrino flux consists in atmospheric neutrinos and downward going atmospheric muons. Atmospheric neutrinos are produced over a large energy range in interactions of primary Cosmic Ray particles (mainly protons) with nuclei in the Earth atmosphere. The resulting hadronic showers contain also short-lived particles like pions and kaons. The main sources of conventional atmospheric neutrinos are their decays

$$
\begin{aligned}
& \pi^{ \pm} \rightarrow \mu^{ \pm}+v_{\mu}\left(\bar{v}_{\mu}\right) \\
& K^{ \pm} \rightarrow \mu^{ \pm}+v_{\mu}\left(\bar{v}_{\mu}\right) \\
& K^{ \pm} \rightarrow e^{ \pm}+v_{e}\left(\bar{v}_{e}\right)+\pi^{0} .
\end{aligned}
$$


The subsequent decay of the muon contributes only marginally to the multi-GeV neutrino flux as most of these muons reach the ground and they are stopped before decaying. The primary Cosmic Rays have a non-thermal $E^{-\gamma}$ spectrum with $\gamma \approx 2.7$. As pions and kaons propagate a certain distance through the atmosphere and lose thereby energy before decaying, the resulting neutrino spectrum is softer with $\gamma \approx 3.7$.

The most recent published measurements of the high energy part of the atmospheric neutrino spectrum comes from the IceCube collaboration [11, 12]. One year of data has been analysed in the IC40 configuration. As many as 18,000 upward going $v_{\mu}$ candidate events have been selected for this analysis. So far only the track signature has been exploited to measure the atmospheric neutrino flux above $100 \mathrm{GeV}$. This has several reasons. First $v_{\mu}$ are more copiously produced than other flavours (see Equation 5.1,5.2) and neutral current interactions have a lower cross section than charged current reactions. Further the effective volume is larger for the muon track signal than for the cascade channel and finally the isolation of a clean upward going event sample is more difficult in the cascade channel, as discussed above.

Two methods have been used to extract the neutrino spectrum from the data, forward folding [11] and regularized unfolding [12]. Both methods give comparable results and they are also consistent with different conventional atmospheric neutrino flux calculations [13, 14]. A distinction between these different flux predictions is not possible within the precision of the measurement. The highest energetic events can be attributed to neutrinos with energies of more than $100 \mathrm{TeV}$, which are thereby the highest energetic neutrino interactions ever detected.

For neutrino energies above $10 \mathrm{TeV}$ the decay of mesons which contain heavy quarks $(\mathrm{c}, \mathrm{b})$ starts to contribute to the atmospheric neutrino flux. As these mesons have typical decay lengths of only few $\mathrm{mm}$, they do not lose energy before they decay and the resulting so-called prompt atmospheric neutrino spectrum follows closely the original Cosmic Ray spectrum, i.e. $\gamma \approx 2.7$. This should be seen as a hardening of the measured spectrum and has been searched for in one of the mentioned IceCube analyses [11]. The predictions for this prompt neutrino flux vary by up to a factor ten $[15,16,17,18,19]$. The largest contribution is obtained in the frame of the Recombination Quark Parton Model [17, 18, 19] (RQPM), a non-perturbative QCD approach. As no hardening of the neutrino spectrum is observed, the RQPM model from [17] can be excluded on a level of $3 \sigma$ in [11]. Further the model from [16] contains free parameters. When choosing these parameters to maximize the prompt neutrino flux, this model can be excluded with $2 \sigma$. The model from [15], which predicts the lowest prompt flux, can instead not be constrained by the current data.

\section{Search for Diffuse Neutrino Fluxes}

For neutrino energies larger than $10 \mathrm{TeV}$ searches are performed for an additional, extraterrestrial component in the measured flux. This component is expected to be harder with $\gamma \approx 2.0$. An upper bound for a diffuse neutrino flux from astrophysical sources has been derived by Waxman and Bahcall (W\&B) [20]. Here it is assumed that the extragalactic Cosmic Ray spectrum for $E>10^{18} \mathrm{eV}$ is produced in sources where protons are magnetically confined to undergo efficiently the photoproduction reaction

$$
p+\gamma \rightarrow \Delta^{+} \rightarrow n+\pi^{+}
$$


The pions decay according to Equation 5.1 and produce neutrinos, whereas the neutrons escape from the acceleration site, decay and produce the observed high energetic Cosmic Ray spectrum. Therefore the predicted neutrino flux is closely related to the observed Cosmic Ray flux above $E>$ $10^{18} \mathrm{eV}$ and should be proportional to $E^{-2}$ over several orders of magnitude. The resulting upper bound, corrected for neutrino oscillations during propagation from the source to Earth (indicated by the factor " $/ 2$ ") and scaled to recent measurements of the highest energetic Cosmic ray flux is shown on Figure 1. Other models try to circumvent the constraints of [20] and predict higher neutrino fluxes. One example [21], already excluded by existing limits, is also shown on Figure 1.

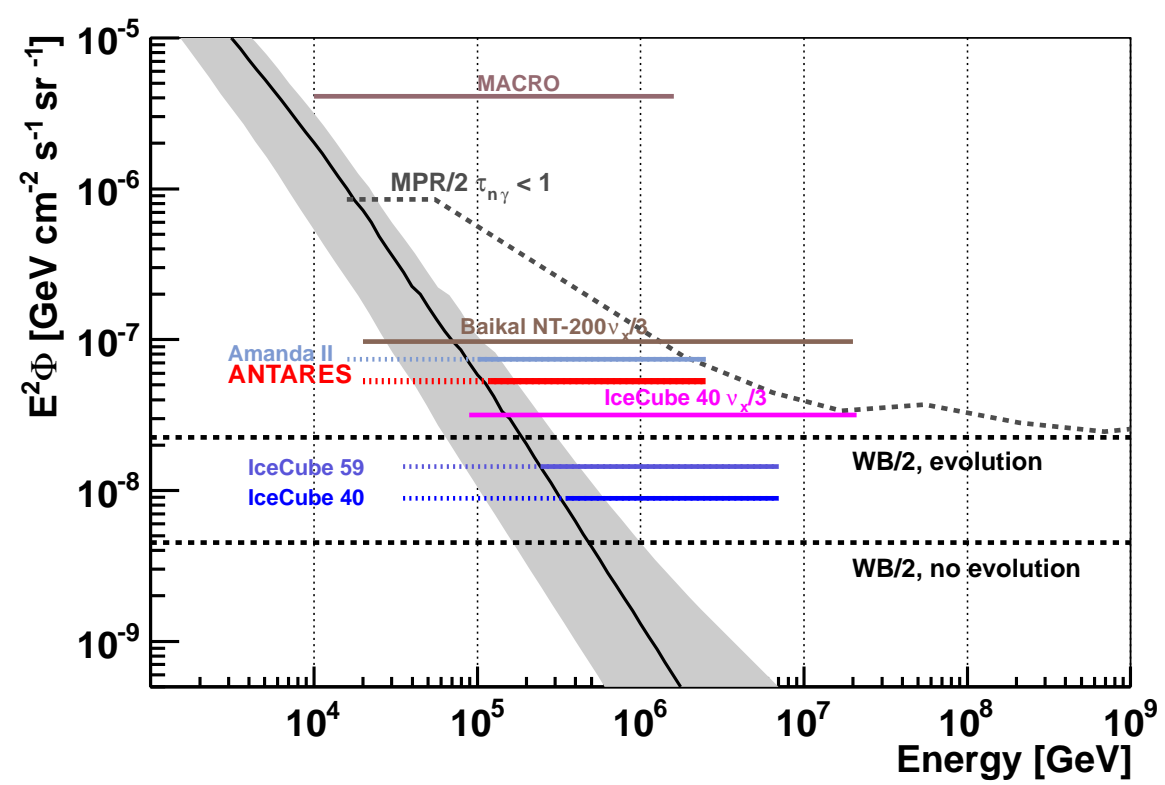

Figure 1: A comparison of published $90 \%$ C.L. upper limits for $E^{-2}$ diffuse neutrino fluxes and theoretical models. For details, see text.

Further a selection of flux limits is shown on Figure 1. All limits are for a C.L. of $90 \%$. They have been derived for $\gamma=2.0$ which results in horizontal lines in the figure. A selection of results from first-generation experiments (Macro [22], Amanda-II [23] and Baikal NT-200 [24]) is compared to the most recent results from ANTARES [25] and IceCube (IC40 [11, 26] and IC59 [27]). The shown limits are for a single neutrino flavours. Most analyses use the muon channel $[22,23,25,11,27]$. Here track reconstruction is used to select a clean sample of upward going neutrino candidates. The energy is estimated by evaluating the "brightness per track length" which correlates with the muon energy loss and therefore the muon energy in the detector. Two analyses have instead exploited the cascade channel $[24,26]$. As such an analysis is sensitive to all neutrino flavours, a scaling factor three (indicated by " $/ 3$ " in the figure) is applied to make the resulting limits compatible to the muon channel limits. The crucial parameter in the cascade analysis is the absolute event brightness, expressed in terms of hit counts or amplitude, which is used as an energy proxy and allows to distinguish the signal from background.

The energy range of the limit lines in Figure 1 indicates the central range in which $90 \%$ of the signal events are expected. For the muon channel, the lowest testable energies are at the level, where the contribution from atmospheric neutrinos becomes stronger. As this contribution is significantly 
lower in the cascade channel (not shown in the figure), the limits derived in this channel extend subsequently to lower energies.

The most recent result has been obtained by analysing 21943 events from 348 days of data taking with the IC59 setup in IceCube [27]. It is interesting to note, that the resulting limit is slightly worse than the limit which had been previously obtained with the smaller IC40 setup [11]. This is due to fluctuations in the high energy tail of the IC59 data set. However the size of the excess is not statistically significant.

\section{Search for Neutrino Point Sources}

The ultimate goal of neutrino telescopes is it to provide a sky map in neutrinos. This requires the identification of individual neutrino sources in the sky and is complementary to the search for a diffuse flux, as described in the previous section. High energy neutrinos can be produced through Fermi acceleration of protons in relativistic shock waves with subsequent hadronic interactions and pion decays. From observation with photons such shock waves are known to exist in various objects in our own Galaxy as well as up to cosmological distances. Various models for neutrino production exist $[28,29,30]$. Promising neutrino point source candidates in our own Galaxy are Supernovae Remnants and Micro Quasars whereas the vicinity of the super-massive black holes in Active Galactic Nuclei and Gamma Ray Bursts are prominent extragalactic candidates.

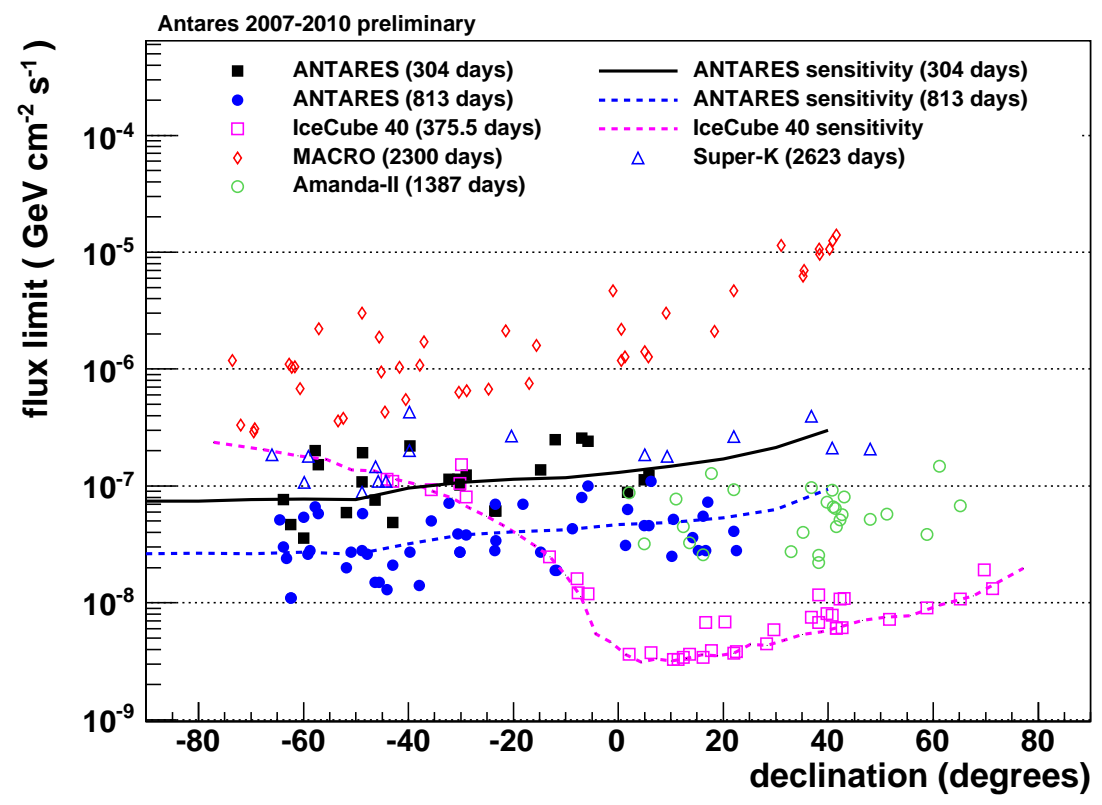

Figure 2: A comparison of published $90 \%$ C.L. upper limits for $E^{-2}$ neutrino fluxes for predefined point source searches. For details, see text.

The first step in the neutrino point source search consists in isolating a suitable set of candidate events. For upgoing events in ANTARES and IceCube the selected events are mostly atmospheric neutrinos. For downgoing events, as analysed in IceCube, the candidate events are high-energetic Cosmic Ray muons. These two event classes follow an almost isotropic distribution on small angular scales. A neutrino point source could be identified as an accumulation of events above 
background around the source. To quantify the discovery potential for a given point source candidate a likelihood ratio method is used. For each event the likelihood is calculated for the hypothesis that it is background (i.e. has an atmospheric origin) or it is signal (i.e. comes from the source). This likelihood takes into account the angular resolution of the events and their energy. The test statistics is the ratio of the two likelihoods, summed over all events in the vicinity of the source candidate. The simulation of many background-only sky maps allows to determine the probability of background fluctuations.

Two methods are used to identify neutrino point sources. In the all-sky-search it is assumed that a source exists somewhere in the sky. Its position is not fixed and it is obtained as the result from a fit to the actual event distribution. When using instead a predefined source list, the source hypothesis is only tested at the positions of the most promising candidates which have been selected a priori by their potential to emit neutrinos and to be seen in the experiment.

None of the searches performed with these methods has identified a neutrino source so far. Figure 2 summarizes the resulting flux limits (assuming an $E^{-2}$ flux from the candidate source) for the predefined source list method as function of declination. All limits are for a C.L. of $90 \%$. Each point is the individual limit for a chosen candidate source. Already existing limits (Macro [31], Superkamiokande [32], Amanda-II [33]) are compared to the most recent results from ANTARES [34, 35] and IceCube (IC40 [36]). The shown limits are all for the muon channel, which has a better sensitivity in the point source search with respect to cascade events due to the superior angular resolution of muon events. For IceCube and ANTARES also the sensitivities are given, which correspond to the median limit which could be derived if many sky maps were considered. It can be seen that the combination of the most recent ANTARES result [35] and IceCube [36] covers the full range in declination (i.e. the whole sky). IceCube is most sensitive in the Northern hemisphere and ANTARES dominates large parts of the Southern hemisphere, which can be easily understood from the geographical location of both experiments. In the mean time IceCube has reported improved limits by about a factor three from a combined analysis of IC40 and IC59 data [27].

\section{Conclusion}

During the last years a wealth of new results have been published on the search for neutrino fluxes from astrophysical sources from both ANTARES and IceCube. Several methods are used to identify such extraterrestrial neutrinos. The most prominent ones are the search for a high energy component to the measured diffuse neutrino flux and by point source searches either from a predefined list of candidate sites for neutrino emission or as a full sky search for a hitherto unknown point in the sky which hides a neutrino source. So far none of these analyses found any excess of events beyond the expected rate of atmospheric neutrino events. The sensitivity of the analyses is already high enough to constrain or exclude various models. For the first time fluxes below the W\&B upper bound [20] are tested.

\section{References}

[1] V. Balkanov et al. [BAIKAL Coll.], In "Salt Lake City 1999, Cosmic ray”, vol. 2 (1999) 176. 
[2] M. Ageron et al., [ANTARES Coll.], Nucl. Instrum. Meth. A656 (2011) 11.

[3] S. Adrian-Martinez et al., [ANTARES Coll.], JINST 7 (2012) T08002.

[4] P. Amram et al., [ANTARES Coll.], Nucl. Instrum. Meth. A484 (2002) 369.

[5] M. Ageron et al., [ANTARES Coll.], Nucl. Instrum. Meth. A578 (2007) 498.

[6] J. A. Aguilar et al., [ANTARES Coll.], Nucl. Instrum. Meth. A622 (2010) 59.

[7] J. A. Aguilar et al., [ANTARES Coll.], Astropart. Phys. 34 (2011) 539.

[8] J. A. Aguilar et al., [ANTARES Coll.], Nucl. Instrum. Meth. A555 (2005) 132.

[9] J. A. Aguilar et al., [ANTARES Coll.], Nucl. Instrum. Meth. A570 (2007) 107.

[10] F. Halzen and S.R. Klein, arXiv:1007.1247.

[11] R. Abbasi et al., [IceCube Coll.], Physical Review D 84 (2011) 082001.

[12] R. Abbasi et al. [IceCube Coll.], Phys. Rev. D 83 (2011) 012001.

[13] G.D. Barr, T.K. Gaisser, P. Lipari, S. Robbins and T. Stanev, Phys. Rev. D 70 (2004) 023006.

[14] M. Honda, T. Kajita, K. Kasahara, S. Midorikawa, and T. Sanuki, Phys. Rev. D 75 (2007) 043006.

[15] A. D. Martin, M. G. Ryskin, and A. M. Stasto, Acta Phys. Pol. B 34 (2003) 3273.

[16] R. Enberg, M.H. Reno, and I. Sarcevic, Phys. Rev. D 78 (2008) 043005.

[17] E. Bugaev et al., Phys. Rev. D 58 (1998) 054001.

[18] G. Fiorentini, A. Naumov, and F. L. Villante, Phys. Lett. B 510 (2001) 173.

[19] C.G.S. Costa, Astropart. Phys. 16 (2001) 193.

[20] E. Waxman and J. Bahcall, Phys. Rev. D 59 (1998) 023002-1;

J. Bahcall, E. Waxman, Phys. Rev, D 64 (2001) 023002-1.

[21] K. Mannheim, R. J. Protheroe, J. P. Rachen, Phys. Rev. D 63 (2000) 023003.

[22] M. Ambrosio et al., [MACRO Coll.], Astrop. Phys. 19 (2003) 1.

[23] A. Achterberg et al., [Amanda Coll.], Phys. Rev. D 76 (2007) 042008.

[24] A.V.Avrorin et al., [Baikal Coll.], Astronomy Letters 35 (2009) 651.

[25] J.A. Aguilar et al., [ANTARES Coll.], Phys. Lett. B 696 (2011) 16.

[26] R. Abbasi et al., [IceCube Coll.], arXiv:1111.2736 [astro-ph.HE].

[27] G.W. Sullivan et al., [IceCube Coll.], Presentation at “Neutrino 2012”, http://neu2012.kek.jp/

[28] F. Halzen and D. Hooper, Rep. Prog. Phys. 65 (2002) 1025.

[29] W. Bednarek, G.F. Burgio and T. Montaruli, New Astron. Rev. 49 (2005) 1.

[30] F.W. Stecker, Phys. Rev. D 72 (2005) 107301.

[31] M. Ambrosio et al., [MACRO Coll.], Astrophysical Journal 546 (2001) 1038.

[32] E. Thrane et al., [SuperK Coll.], Astrophysical Journal 704 (2009) 503.

[33] R. Abbasi et al., [Amanda Coll.], Phys. Rev. D 79 (2009) 062001.

[34] S. Adrian-Martinez et al., [ANTARES Coll.], Astrophys. J. Lett. 743 (2011) L14.

[35] S. Adrian-Martinez et al., [ANTARES Coll.], Astrophysical Journal 760 (2012) 53.

[36] R. Abbasi et al. [IceCube Coll.], Astrophysical Journal 732 (2011) 18. 